\title{
Investigating the Merger Origin of Early-type Galaxies using Ultra-deep Optical Images
}

\author{
P.-A. Duc ${ }^{1}$ J.-C. Cuillandre,${ }^{2}$ K. Alatalo,${ }^{3}$ L. Blitz,${ }^{3}$ M. Bois,${ }^{4}$ \\ F. Bournaud, ${ }^{1}$ M. Bureau, ${ }^{5}$ M. Cappellari,${ }^{5}$ P. Côté,${ }^{6}$ R. L. Davies,${ }^{5}$ \\ T. A. Davis, ${ }^{5}$ P. T. de Zeeuw ${ }^{7,8}$ E. Emsellem, ${ }^{7,4}$ L. Ferrarese, ${ }^{6}$ \\ E. Ferriere, ${ }^{1}$ S. Gwyn, ${ }^{6}$ S. Khochfar,${ }^{9}$ D. Krajnovic,${ }^{7}$ H. Kuntschner, ${ }^{7}$ \\ P.-Y. Lablanche,${ }^{4}$ L. MacArthur,${ }^{6}$ R. M. McDermid,${ }^{10}$ \\ L. Michel-Dansac, ${ }^{4}$ R. Morganti, ${ }^{11}$ T. Naab, ${ }^{12}$ T. Oosterloo, ${ }^{11}$ \\ M. Sarzi ${ }^{13}$ N. Scott, ${ }^{5}$ P. Serra, ${ }^{11}$ A. Weijmans ${ }^{14}$ and L. M. Young ${ }^{15}$ \\ ${ }^{1}$ AIM Paris-Saclay, France $;{ }^{2}$ CFHT, USA $;{ }^{3}$ University of California, Berkeley, USA; \\ ${ }^{4}$ Observatoire de Lyon, France; ${ }^{5}$ University of Oxford, UK; ${ }^{6}$ Herzberg Institute of \\ Astrophysics, Victoria, Canada; ${ }^{7}$ ESO, Garching, Germany; ${ }^{8}$ Leiden University, The \\ Netherlands; ${ }^{9} \mathrm{MPE}$, Garching, Germany; ${ }^{10}$ Gemini Observatory, Hilo, USA; ${ }^{11}$ ASTRON, \\ Dwingeloo, The Netherlands; ${ }^{12}$ MPI, Garching, Germany; ${ }^{13}$ University of Hertfordshire, \\ Hatfield, UK; ${ }^{14}$ Dunlap Institute for Astronomy \& Astrophysics, University of Toronto, \\ Canada $;{ }^{15}$ New Mexico Tech, Socorro, USA
}

\begin{abstract}
The mass assembly of galaxies leaves various imprints on their surroundings, such as shells, streams and tidal tails. The frequency and properties of these fine structures depend on the mechanism driving the mass assembly: e.g. a monolithic collapse, rapid cold-gas accretion followed by violent disk instabilities, minor mergers or major dry/wet mergers. Therefore, by studying the outskirts of galaxies, one can learn about their main formation mechanism. I present here our on-going work to characterize the outskirts of Early-Type Galaxies (ETGs), which are powerful probes at low redshift of the hierarchical mass assembly of galaxies. This work relies on ultra-deep optical images obtained at CFHT with the wide-field of view MegaCam camera of field and cluster ETGs obtained as part of the ATLAS ${ }^{3 \mathrm{D}}$ and NGVS projects. State of the art numerical simulations are used to interpret the data. The images reveal a wealth of unknown faint structures at levels as faint as $29 \mathrm{mag} \operatorname{arcsec}^{-2}$ in the g-band. Initial results for two galaxies are presented here.
\end{abstract}

Keywords. galaxies: formation, galaxies: evolution, galaxies: interactions, galaxies: elliptical and lenticular, $\mathrm{cD}$

\section{Introduction}

There is a rather large consensus to ascribe to galaxy mergers a prominent role in the formation of Early-Type Galaxies, especially the most massive ones. For almost 40 years, numerical simulations and observations have supported this hypothesis. The debate is however still very active on the details of this merger-based mass assembly: is it primarily due to minor/major, dry/wet mergers? When did the merging events occur, and in which types of environment? Study of their internal properties, in particular their kinematics, shows that the properties of ETGs change along the luminosity function, with a general trend for galaxies to transition from slow to fast rotators, while peculiar objects can show distinct features such as Kinematically Decoupled Cores (see the review by E. Emsellem in this volume, and references therein). Are mergers able to reproduce the full variety of observed properties? Alternatively, it has been proposed that a fraction of 


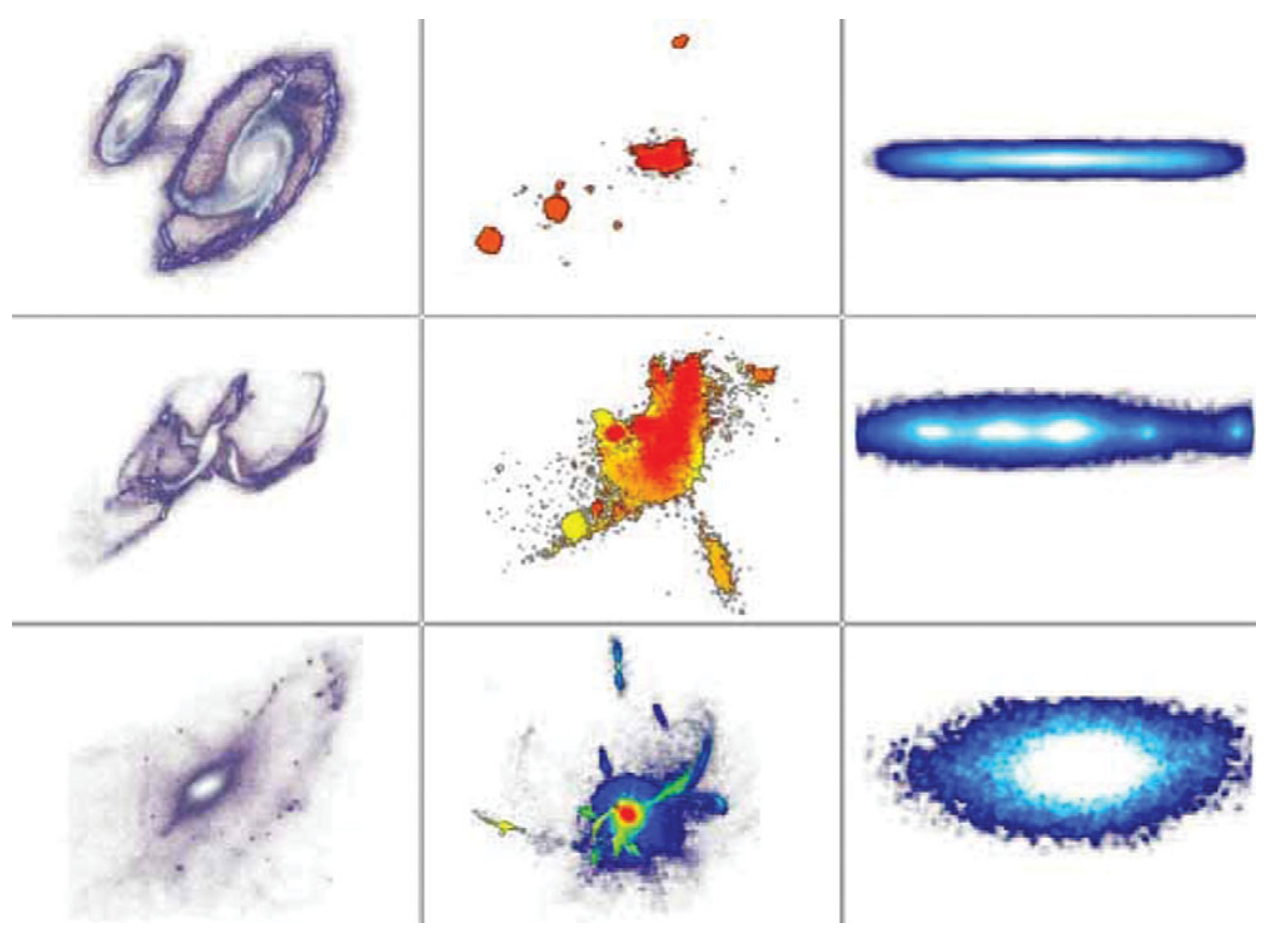

Figure 1. Predictions from numerical simulations of the frequency, colors and shapes of fine structures for three mass-assembly scenarios. Time sequences are shown for a major merger (left col., Bournaud et al., 2008), multiple collisions (middle col., from Martig et al., 2009) and evolution of an unstable disk (right col., Elmegreen et al., 2008).

ETGs, especially those with high ellipticity, might have formed at high redshift by secular evolution, i.e. from the interaction, migration and merging of massive condensations formed in highly turbulent disks, supplied by cold gas flows (Elmegreen et al., 2008).

All these processes produce remnants that have a luminosity profile characterized by a high Sersic index, but with very different morphologies in the outermost regions. While the secular model does not produce any fine structure (Fig.1, right), the imprint of (multiple) minor mergers are shells and narrow stellar streams (Fig.1, middle) and that of major mergers between spirals are prominent, long, tidal tails (Fig.1, left). Thus, by studying the properties of collisional debris, one might be able to reconstruct the mass assembly of their progenitors. This method must, however, address the following issues (1) debris has a very low-surface brightness, usually below 26 mag $\operatorname{arcsec}^{-2}$ in the $\mathrm{g}$ band (2) debris fades with age - it might be used as a clock for age-dating merger events - or may be dispersed in their environment: the memory of its origin is then lost (3) galaxies may be formed by hybrid processes. Current detailed numerical simulations done in cosmological context precisely address these issues and are now capable of relating the merging/accretion history of the simulated galaxies with the number, shape, brightness and ages of the fine structures present at the end of their evolution.

\section{Sample, observations and data processing}

The ultra-deep images presented here were obtained with the large field-of-view MegaCam camera installed on the Canada-France-Hawaii Telescope (CFHT). They were taken as part of two large projects: 


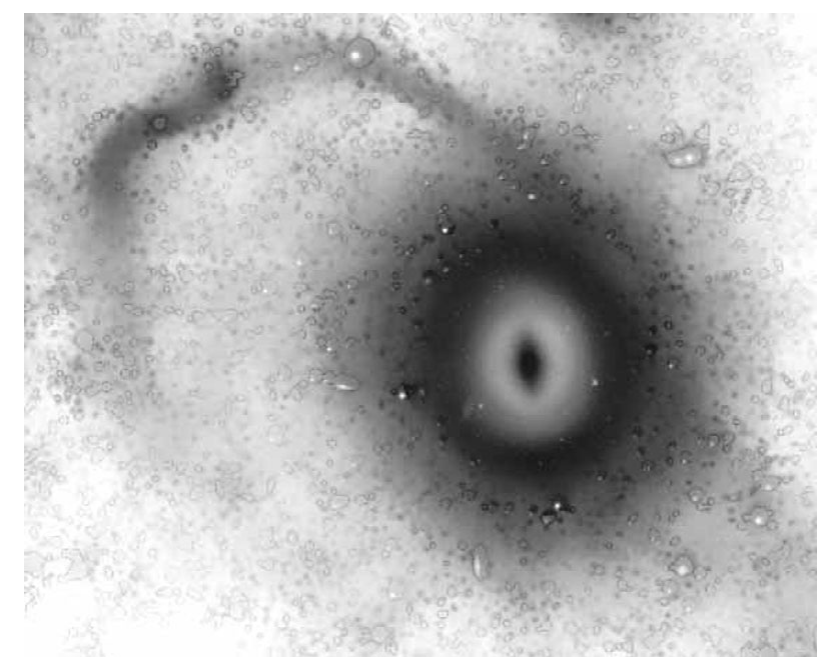

Figure 2. A satellite galaxy torn away by tidal forces, around an early-type galaxy in the Virgo Cluster. The SDSS image of the galaxy (showing a strong bar) is superimposed on an ultra-deep MegaCam image extracted from the NGVS. The foreground stars have been subtracted.

- $\operatorname{ATLAS}^{3 \mathrm{D}} \dagger$, a multi-wavelength survey of a complete sample of 260 nearby earlytype galaxies within $42 \mathrm{Mpc}$, complemented by semi-analytical and numerical models (Cappellari et al., 2011). A sub-sample of approximately 100 ETGs located in the field and in groups were selected for follow-up deep optical imaging in the g' and r' bands.

- the Next Generation Virgo Cluster Survey (NGVS) $\ddagger$, which maps the entire Virgo Cluster area in the $\mathrm{u}^{*}, \mathrm{~g}^{\prime}, \mathrm{r}^{\prime}, \mathrm{i}^{\prime}$ and z' bands (Ferrarese et al., 2011, in prep.). Within the 104 square-degrees covered by the NGVS, there are about 50 ETGs that are part of the ATLAS $^{3 \mathrm{D}}$ sample.

These two surveys reach the same surface brightness limit: about $29 \mathrm{mag} \operatorname{arcsec}^{-2}$ in the g-band (about $2.5 \mathrm{mag}$ deeper than the SDSS). Such a sensitivity could only be achieved by using specific observing and data-reduction procedures. Basically, each observation consisted of multiple exposures dithered with a very large pattern (between 7' and 70') so as to minimize sky background variations. The total exposure time varied between 0.5 and 2 hours depending on the band. The data were processed at CFHT with the Elixir Low Surface Brightness arm of the MegaCam pipeline at CFHT (Cuillandre et al. 2011, in prep.).

\section{Discovery of new fine structures around ETGs}

The unprecedented depth achieved by these surveys introduces us to a new imaging regime, full of faint foreground stars, halos of bright stars, extended, scattered light from galactic cirrus, distant background galaxies and clusters .... and previously unknown stellar streams associated to the early-types galaxies. Two examples of newly discovered extended stellar structures are presented here. Elongated, narrow streams from a disrupted satellite, wrapping around the host galaxy are visible in Fig. 2. This is the first step of a minor merger. Fig. 3 discloses prominent, long, tidal tails sticking out of what was so far considered as a massive, relaxed, elliptical, classified as a slow rotator, with the ATLAS ${ }^{3 D}$ definition (Emsellem et al., 2011). Duc et al. (2011) argue that these

$\dagger$ http://www-astro.physics.ox.ac.uk/atlas3d

$\ddagger$ https://www.astrosci.ca/NGVS/The_Next_Generation_Virgo_Cluster_Survey 


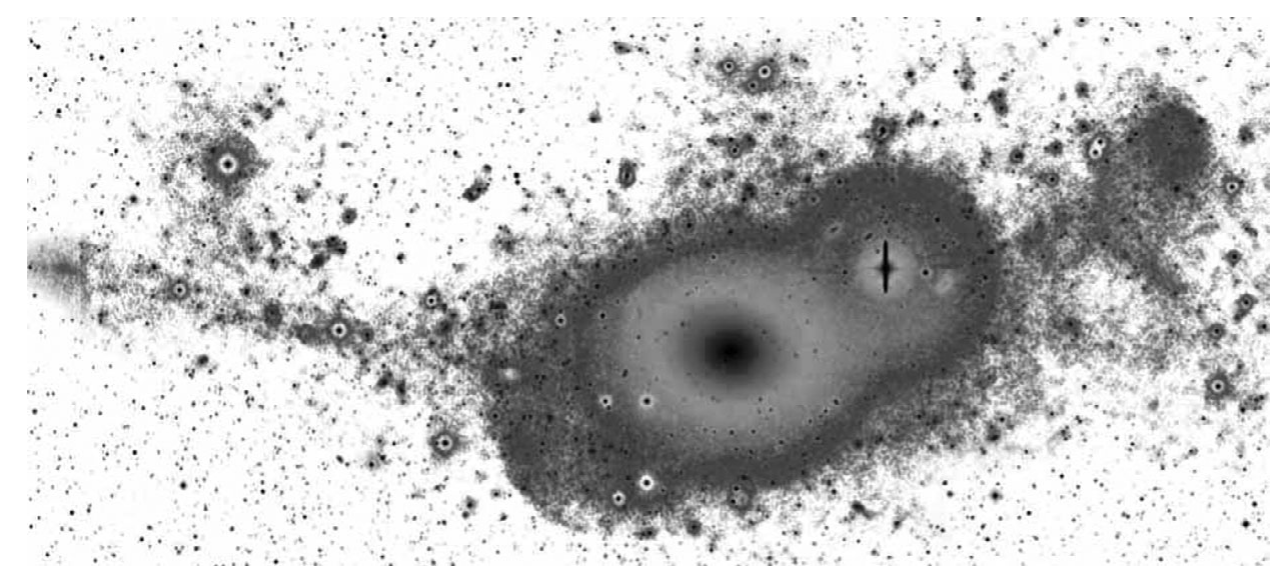

Figure 3. A massive, round, slow rotator in the ATLAS ${ }^{3 \mathrm{D}}$ sample of nearby Early Type Galaxies. The MegaCam image discloses a series of narrow tails and shells that suggest a rather recent merger. The longest tail has a size of at least $160 \mathrm{kpc}$. The black stain at the center of the ETG shows the extent of the galaxy as previously known from the SDSS (Duc et al., 2011)

features are likely collisional debris of a relatively recent major merger that occurred 2 to 5 Gyr ago. The detection of HI gas along some blue condensations in the tail - in fact mature Tidal Dwarf Galaxies - suggests that the progenitors were gas-rich as well. Without the support of ultra-deep images, such clouds might have been interpreted as signs of on-going gas accretion from cold flows (see review by van Gorkom in this volume for possible examples of such clouds around nearby galaxies). The formation of a massive, round, ETG by a wet merger that occurred below a redshift of 0.5 is at odds with some predictions from semi-analytical models and numerical simulations: indeed, the formation of such galaxies seems to require a long process involving a high-z major merger followed by multiple minor mergers.

These initial results illustrate the potential of ultra-deep optical images to reconstruct the mass assembly of individual galaxies and trace their ancestors. Furthermore, the ATLAS $^{3 D}$ /NGVS MegaCam images and follow-up multi-wavelength surveys will provide statistics on the number and properties of the fine structures around galaxies, and thus allow to investigate how the main mechanism driving the mass assembly may vary as a function of their morphological type and large-scale environment.

\section{References}

Bournaud, F., Duc, P.-A., \& Emsellem, E. 2008, MNRAS, 389, L8

Cappellari, M. et al. 2011, MNRAS, in press (arXiv:1012.1551)

Duc, P-A., et al. 2011, MNRAS, submitted

Elmegreen, B. G., \& Bournaud, F. and Elmegreen, D. M. 2008, ApJ, 688, 67

Emsellem, E.., et al.. 2011, MNRAS, submitted

Martig, M. et al. 2009, ApJ, 707, 250 